\title{
Recent Progress Towards Centimetric Spatial Resolution in Distributed Fibre Sensing
}

\author{
Luc Thévenaz*, Stella Foaleng-Mafang, Kwang-Yong Song ${ }^{\text {a }}$, Sanghoon Chin, \\ Jean-Charles Beugnot, Nikolay Primerov, Moshe Tur ${ }^{\mathrm{b}}$ \\ EPFL Swiss Federal Institute of Technology, Institute of Electrical Engineering, STI-GR-SCI-LT, \\ Station 11, 1015 Lausanne, Switzerland
}

\begin{abstract}
Optical fibre sensors based on stimulated Brillouin scattering have now clearly demonstrated their excellent capability for long-range distributed strain and temperature measurements. The fibre is used as sensing element and a value for temperature and/or strain can be obtained from any point along the fibre. While classical configurations have practically a spatial resolution limited by the phonon lifetime to 1 meter, novel approaches have been demonstrated these past years that can overcome this limit. This can be achieved either by the prior activation of the acoustic wave by a long lasting pre-pumping signal, leading to the optimized configuration using Brillouin echoes, or by probing a classically generated steady acoustic wave using a ultra-short pulse propagating in the orthogonal polarization of a highly birefringent fibre. These novel configurations can offer spatial resolutions in the centimetre range, while preserving the full accuracy on the determination of temperature and strain.
\end{abstract}

Keywords: Fibre optics, optical fibre sensor, distributed fibre sensor, stimulated Brillouin scattering, nonlinear optics.

\section{INTRODUCTION}

Distributed Brillouin sensing was first proposed in the late 1980's as an alternative technique to the classical OTDR to measure local attenuation along an optical fibre ${ }^{1}$. It turned out rapidly that it had many more potentialities for sensing, since Brillouin scattering is intrinsically very sensitive to temperature and the deformations experienced by the sensing fibre ${ }^{2,3}$. This comes from the property that Brillouin scattering must satisfy a very strict phase matching condition, making the interaction observable as a resonance spectrally spreading over a very narrow band (typ. $27 \mathrm{MHz}$ at a wavelength of $1550 \mathrm{~nm}$ ). For a spontaneous scattering the backscattered light will present a sharp spectrum peaking at a frequency shifted from the pump by the quantity ${ }^{4}$ :

$$
v_{B}=\frac{2 n V_{a}}{\lambda_{o}}
$$

commonly named Brillouin frequency shift, where $n$ represents the effective refractive index of the propagating mode, $V_{a}$ the acoustic velocity in the fibre $(\sim 5800 \mathrm{~m} / \mathrm{s})$ and $\lambda_{o}$ the vacuum wavelength of the incident light.

For a stimulated scattering a weak signal wave will experience a sharp gain if it spectrally positioned at a frequency shifted by $v_{B}$ below a strong pump propagating in the opposite direction through the fibre.

As expressed in (1) the Brillouin frequency shift $v_{B}$ - that is typically $10-11 \mathrm{GHz}$ in a silica fibre at $1550 \mathrm{~nm}$ - is very dependent on the acoustic velocity and any change of this velocity will be observed as a spectral shift of the resonance. In a solid the theory of elasticity gives the following general expression for the acoustic velocity ${ }^{4}$ :

$$
V_{a}=\sqrt{\frac{K}{\rho}} \quad \text { with } K \text { the bulk modulus and } \rho \text { the material density. }
$$

\footnotetext{
*Luc.Thevenaz@EPFL.CH; phone +41 21693 4774; fax +41 21693 4660; epfl.ch

${ }^{a}$ Permanent address: Department of Physics, Chung-Ang University, Seoul 156-756, Korea

${ }^{\mathrm{b}}$ Permanent address: Faculty of Engineering, Tel-Aviv University, Tel Aviv 69978, Israel
} 
The density of the material $\rho$ is temperature-dependent as a result of the thermal expansion, so that the peak frequency of the interaction is observed to change with the temperature. Any deformation experienced by the fibre will also impact on its density $\rho$, and this property is particularly exploited to use the fibre as a distributed strain gauge, by observing the shift of the resonance when the fibre is elongated or compressed.

So temperature and strain can be evaluated using Brillouin scattering by determining the frequency difference between pump and signal that gives the maximum interaction. It is remarkable that the relationship between these two quantities and the Brillouin shift $v_{B}$ is very linear under standard conditions (temperature between -100 and $+500^{\circ} \mathrm{C}$, strain $\pm 1 \%$ ) ${ }^{5}$.

From the point of view of the measuring potentialities Brillouin-based techniques bring the following advantages over other distributed techniques:

- The technique makes use of standard low-loss single-mode optical fibres offering several tens of kilometres of distance range and a compatibility with telecommunication components.

- It is a frequency-based technique as opposed to Raman techniques which are intensity-based. Brillouin techniques are consequently inherently more accurate and more stable on the long term, since intensity-based techniques suffer from a higher sensitivity to drifts and from a potential biasing by any step loss.

- Since the information is not a consequence of the background thermal activation experimental cofigurations based on stimulated Brillouin scattering can be exploited, leading to a much greater intensity of the scattering mechanism and consequently a more acceptable signal-to-noise ratio.

Instruments based on spontaneous Brillouin for Time Domain Reflectometry (BOTDR) ${ }^{6}$ require only one access point to the test fibre to launch the pump wave, while those using SBS for Brillouin Analysis (BOTDA) must access both ends of the test fibre to launch the pump pulse and $\mathrm{CW}$ signal ${ }^{3,7,8}$. Both types of instruments offer good accuracy for temperature and strain measurements, albeit at spatial resolutions not better than $\sim 1 \mathrm{~m}^{9}$. The reason comes from the fact that the observed Brillouin spectrum broadens as the pulse width decreases, since the spectral distribution of the effective gain is given by the convolution between the pulse spectrum, broader for shorter pulses, and the natural Brillouin gain spectrum $\left(\sim 30 \mathrm{MHz}\right.$ in silica fibres at $\lambda_{p}=1550 \mathrm{~nm}$, determined by the phonon lifetime $\left.\tau_{A} \cong 6 \mathrm{~ns}\right){ }^{10}$. This broadening leads to a reduced peak gain and uncertainties in the determination of the Brillouin shift $v_{B}$, and consequently to a reduced accuracy in the measurement of temperature and strain at submetric spatial resolutions. A natural limit is given by the situation when pump pulse and Brillouin gain spectra show comparable bandwidth, corresponding to a pump pulse showing a duration equal to a double phonon lifetime $2 \times \tau_{A} \cong 12 \mathrm{~ns}$, or $\sim 1$ meter spatial resolution.

However, modern advanced applications require a substantial step towards better spatial resolution, while preserving temperature/strain precision over a long range. For this purpose stimulated Brillouin scattering offers the possibility of very innovative schemes, as will be shown hereafter.

The record spatial resolution is currently obtained by a correlation technique proposed in 2000 by Hotate et al from the University of Tokyo ${ }^{11,12}$. In this elegant alternative technique the correlation between two continuous lightwaves is controlled. This way Brillouin scattering can be generated locally along the fibre and the use of a pulsed lightwave is no longer required. Frequency modulation (FM) is the technique that was implemented to create local correlations and centimetric spatial resolutions can be routinely obtained, however over a limited distance.

Nearly equivalent spatial resolutions can nevertheless be obtained today using pure time-domain techniques over kilometric ranges that will be presented in the following sections.

\section{BRILLOUIN ECHO DISTRIBUTED SENSING (BEDS)}

The commonly accepted opinion that the spatial resolution of a pulse-based Brillouin sensor is limited to $1 \mathrm{~m}$ was seriously questioned when Bao et al observed an unexpected narrowing of the Brillouin gain spectrum down to its natural linewidth when pulses turned shorter than the phonon lifetime $(6 \mathrm{~ns})^{13}$. This breakthrough opened the incredible perspective of performing high spatial resolution measurement while maintaining a sharp Brillouin resonance.

It is now unanimously accepted by all specialists that this special behaviour results from a pre-excitation of the acoustic wave through the presence of a continuous background pump ${ }^{14}$. This was originally observed by using a modulator with a poor off-state extinction ratio to form the pump pulses. 
Basically, the observation of this effect depends on the pre-existence of an important acoustic wave in the fibre medium vibrating at the exact Brillouin frequency resonance. This acoustic wave is conveniently generated through stimulated Brillouin scattering using a continuous wave at the probe frequency and another continuous wave (or a long pulse) at the pump frequency. Among the 3 waves involved in the interaction, the 2 optical waves can experience very fast changes in their amplitude and phase, while the acoustic wave presents a highly inertial behaviour and requires a typical time equal to a multiple of its 6 ns lifetime to adapt to a new situation. For instance, if the pump is suddenly turned off, the acoustic wave will gradually decay and will still exist during the typical duration of its lifetime, despite the absence of stimulated interaction.

This inertial property is used to change very briefly amplitude or phase of the pump, during a time $T$ so short that the acoustic wave does not experience a notable change ( $T<<$ phonon lifetime). This way the pump will be reflected by the acoustic wave, but under transient conditions that do not correspond to those required by amplitude and phase matching. This will cause an abrupt change of the steady state amplification of the probe that can be observed by a fast detection scheme. The pre-existence of the acoustic wave is essential and, since it is stimulated by continuous optical waves, a response will be observed only over the natural local Brillouin gain spectrum along the fibre.

To highlight the similarity with spin echoes, this new concept is named Brillouin echo because it turns out to be formally very similar to the description of the interaction of nuclear spins with magnetic fields in nuclear magnetic resonance.

The Brillouin echo can be observed essentially with 3 different pump pulse coding, detailed here below.

1. Bright pulse: the effect was first observed using this configuration ${ }^{13}$. The finite extinction ratio of the electro-optic modulator let a continuous flow of pump light activate the acoustic wave, giving constant background amplification on the probe (dark topping). The additional pump light in the pulse is reflected and its effect is observed as an additional amplification. This configuration is the less efficient if the peak power of the pump is fixed.

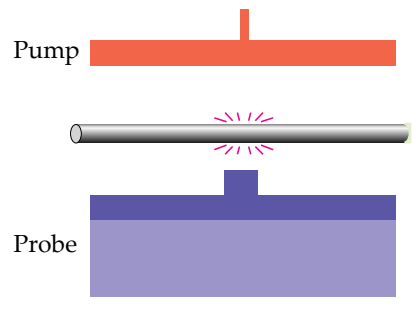

2. Dark pulse: The pump is turned off during a short time, so that no light is reflected and the constant background amplification is stopped ${ }^{15}$. The acoustic wave keeps vibrating during the dark pulse and the background amplification is restored when the pump light is on again. The effect of the dark pulse is observed as a "negative" gain or an apparent loss (actually it is an absence of gain).

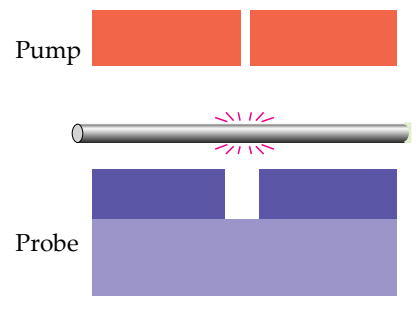

3. Phase pulse: A $\pi$-phase shift is applied on the pump for a short time, so that the reflected light interferes destructively with the signal, equivalent to a Brillouin loss process ${ }^{16,17}$. The response is identical to the dark pulse, but is twice larger for an identical pump power and is thus the most efficient ${ }^{18}$.

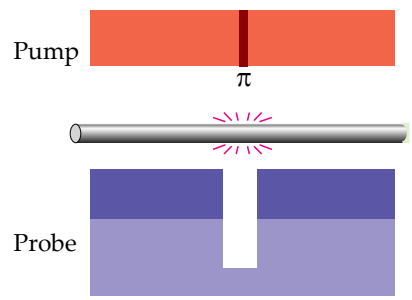

The set-up implementation is very similar to a BOTDA configuration, as shown in Figure 1. For the 2 first situations, the pump pulses are also shaped by an intensity electro-optic modulator that is simply electrically biased differently. In the case of the $\pi$-phase pulse, a phase modulator substitutes for the intensity modulator. 


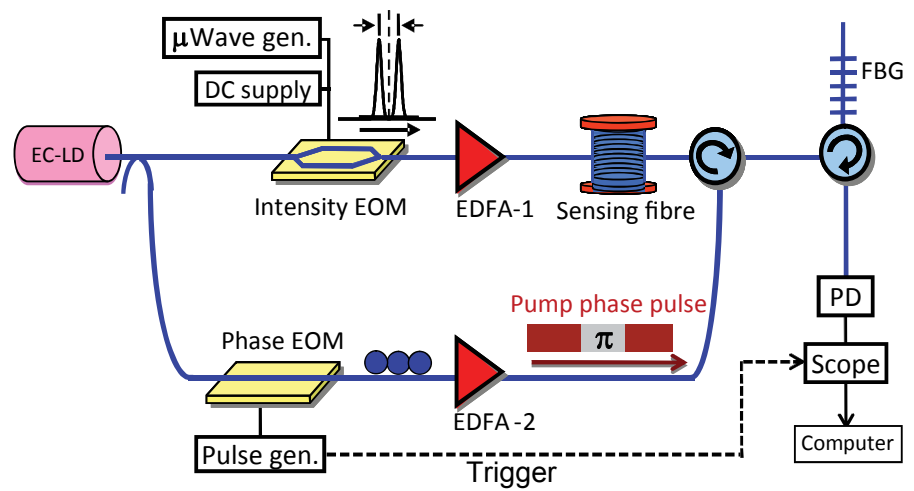

Figure 1. Block diagram of a BEDS set-up based on a $\pi$-phase modulation of the pump.

The most important problem faced by such a technique is the fact that the acoustic wave is partially decaying during the pulse duration. This creates a second attenuated echo when the pump is restored to its original state after the pulse, but this echo extends over a duration given by the acoustic lifetime and thus creates a ghost response over the subsequent measured points ${ }^{18}$. Two solutions have been identified to attenuate or even to totally suppress the impact of the $2^{\text {nd }}$ echo:

1. By deconvolving numerically the time traces: the impulse response of any BEDS sensor has been analytically determined and in particular the contribution of the $2^{\text {nd }}$ echo to this impulse response can be unambiguously extracted ${ }^{18}$. This contribution can then be removed from the time traces by a simple step-by-step single pass algorithm.

2. By turning off the pump immediately after the pump phase pulse: no more light from the pump can be reflected after the pulse end and no trailing light is present. This solution was successfully tested ${ }^{17}$ for a phase pulse in a differential measurement configuration by subtracting the traces obtained with the phase pulse turned on and off, successively, and was recently adapted for a bright pulse ${ }^{19}$.

Figure 2 (left) shows the distribution of Brillouin amplification over the last $40 \mathrm{~m}$ of a $4.57 \mathrm{~km}$ fibre, obtained using a $500 \mathrm{ps} \pi$-phase pulse in the differential measurement technique described above. A $5 \mathrm{~cm}$ segment showing a distinct Brillouin shift can be clearly identified and measured even after several kilometres of fibre, as shown in the right graph of Figure 2. Recently the range could be even extended to $10 \mathrm{~km}$, reaching the tremendous record of 200 '000 independently resolved points. The accuracy on the Brillouin shift determination remains comparable to a standard BOTDA technique, since no significant broadening of the measured Brillouin spectrum is observed.
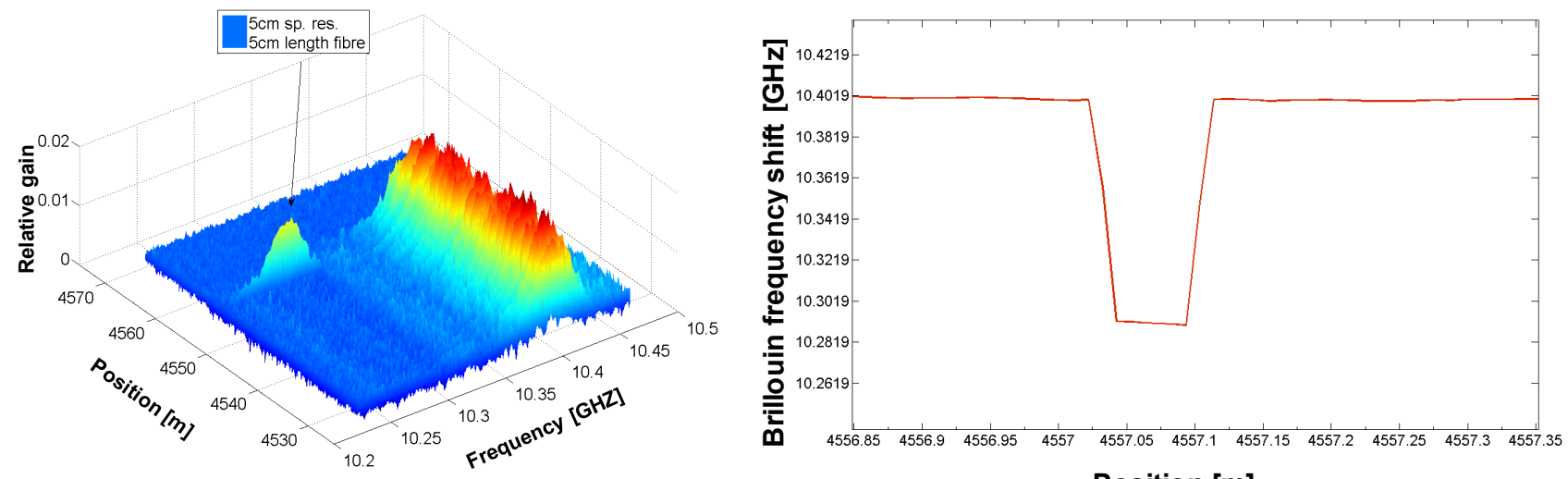

Position [m]

Figure 2. Left: 3D view of the signal amplification obtained using the BEDS technique in a differential configuration with $500 \mathrm{ps} \pi$-phase shifts on the pump. Here are shown the 40 last meters of a $4.57 \mathrm{~km}$ fibre, where a $5 \mathrm{~cm}$ segment showing a distinct Brillouin shift is inserted.

Right: distribution of the Brillouin frequency shift at the position of this segment obtained after processing, showing that the segment is clearly resolved (horizontal axis limited to a $1.5 \mathrm{~m}$ range). 


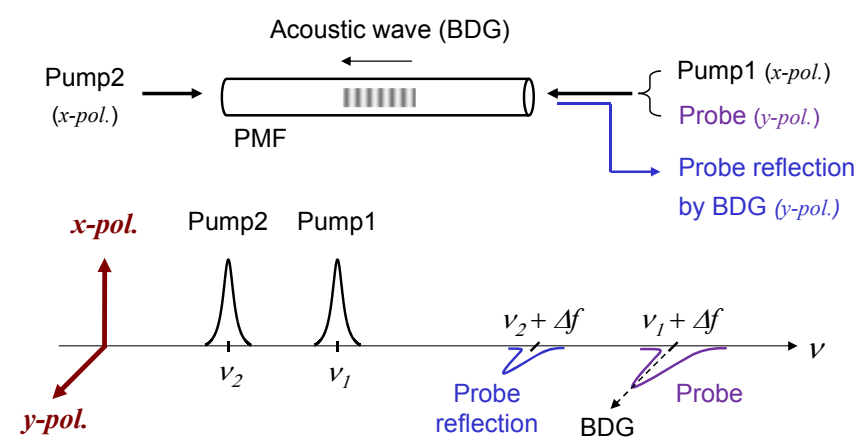

Figure 3. Description of the concept for the generation and the reading of Brillouin dynamic grating.

\section{DYNAMIC BRILLOUIN GRATING DISTRIBUTED SENSING (DBG-DS)}

In recent studies ${ }^{20-22}$, the concept of Brillouin dynamic grating (BDG) has been newly implemented in polarizationmaintaining fibres (PMF), where acoustic waves generated during the process of stimulated Brillouin scattering (SBS) by optical waves (pump waves) in one polarization are used to reflect an orthogonally-polarized wave (probe wave) at a different optical frequency from the pump. As shown in the conceptual scheme in Figure 3, the frequency separation $\Delta f$ between the pump and the probe waves in the BDG operation is determined by the birefringence of the PMF and lies in the several ten's of GHz in usual cases. As a potential application, a novel type of birefringence-based distributed sensor has been proposed using the BDG with an order-of-magnitude higher sensitivity in the temperature measurement compared to ordinary Brillouin sensors ${ }^{22-24}$ and even offering a full and well conditioned discrimination between strain and temperature ${ }^{25}$.

BDG can also be applied to enhance the spatial resolution of an ordinary Brillouin optical time-domain analysis (BOTDA) system by replacing the Brillouin probe with the reflection from the BDG. It is possible this way to acquire a narrowband Brillouin gain spectrum (BGS) with a broadband pulse using the BDG, and measurements with a $1 \mathrm{~cm}$ spatial resolution have been demonstrated experimentally, which is the best result ever reported using a time-domain Brillouin sensor ${ }^{26}$.

Figure 4 shows the principle of the BDG distributed sensing where the pump waves (Pump 1 and Pump 2) in the $x$ polarization and a probe pulse in the $y$-polarization are applied for the generation of the BDG (i.e. acoustic wave) and its readout, respectively. In this scheme, a relatively long pulse (30 ns) for Pump 1 is used to maintain a narrowband Brillouin gain, meanwhile adopt a ultra-short probe pulse $(\sim 110 \mathrm{ps})$ to realize a high-resolution acquisition of the local Brillouin gain spectrum.

When the broadband probe pulse is reflected by the local BDG, the reflectance, which is determined by the spectral overlap between the BDG and the probe pulse, is proportional to the strength of the BDG. It means that the time trace of the probe reflection shows the local strength of the BDG, which depends on the Brillouin gain in the process of SBS between the two pump waves. Thus, the shape of the BGS can be retrieved from the probe reflection by sweeping the frequency offset between Pump 1 and Pump 2. Since the spatial resolution of this measurement is determined by the duration of the probe pulse while the bandwidth of the Brillouin gain is related to the duration of Pump 1, a high spatial resolution can be achieved while keeping a narrow effective Brillouin gain spectrum. It must be pointed out that the probe frequency is not scanned, since its spectrum covers a much broader spectrum than any reasonable change of the BDG frequency due to environment, as shown in Figure 4.

A typical implementation of a BDG-based is shown in Figure 5. It must be pointed out that Pump 1 must be preferably pulsed to avoid any depletion and an excessive gain for Pump 2, nonetheless with a duration far exceeding the acoustic lifetime (>10 ns). A measurement of a $1.5 \mathrm{~cm}$ hot spot is presented in Figure 6, obtained using $110 \mathrm{ps}$ probe pulse. The smooth transitions are not due to a limitation in the resolving power, but to the natural thermal transfer along the optical fibre that can be observed in this situation. 


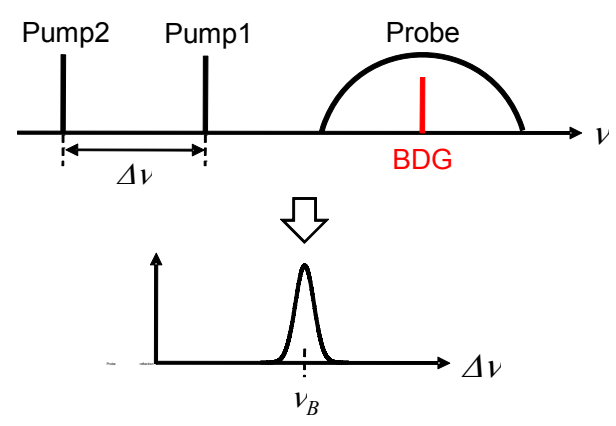

Figure 4. Acquisition of a local Brillouin gain spectrum with a short pulse in a BDG-based distributed sensing system through the BDG-strength variation.

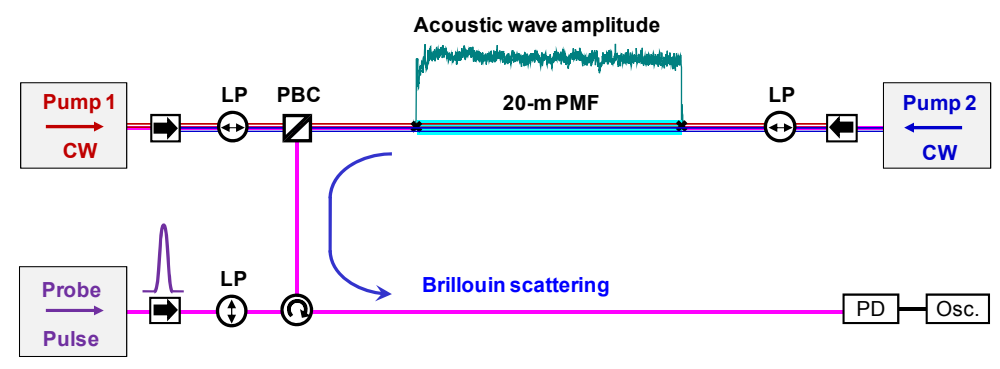

Figure 5. Block diagram of a typical implementation of the BDGDS set-up. Pump 1 and Pump 2 can be generated from the same laser using a similar technique as in a BOTDA system, based on the modulation sideband. LP: linear polarizer, PBC: polarization beam combiner, PD: photodiode.

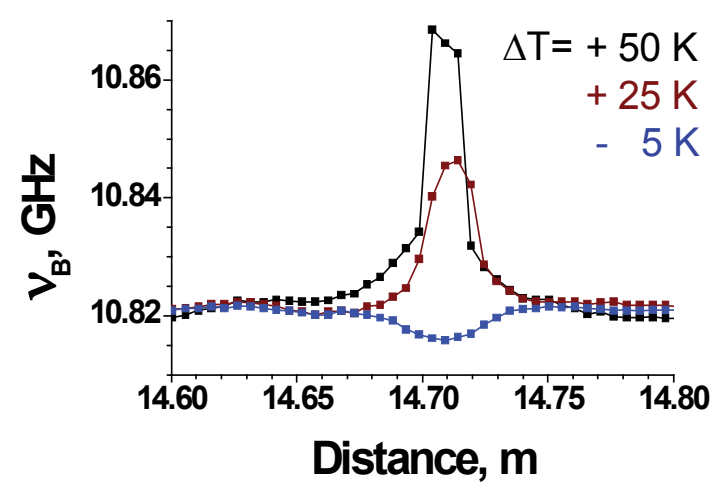

Figure 6. Measurement of a $1.5 \mathrm{~cm}$ spot placed at different temperatures with a DBG-DS system using 110 ps probe pulses.

The temperature difference with respect to the environment is indicated.

This shows the huge progresses achieved these past few years in Brillouin time-distributed sensing, showing the possibility to resolve events with a spatial resolution in the centimetre range This is fully comparable to point sensors such as fibre Bragg gratings, however showing the great flexibility of a fully continuous distributed sensing, equivalent to many thousands of distinct point sensors and requiring no special fibre preparation. This will certainly cause a significant change in the appreciation of this type of sensors, by broadening the field of applications to small and medium-size structures.

\section{ACKNOWLEDGMENT}

The authors are particularly grateful for their support to the company Omnisens and to partners in the COST Action 299 "FiDES".

\section{REFERENCES}

1. T. Horiguchi and M. Tateda, "Optical-fiber-attenuation investigation using stimulated Brillouin scattering between a pulse and a continuous wave," Opt. Lett. 14(8), 408-410 (1989)

2. T. Horiguchi, T. Kurashima and M. Tateda, "A technique to measure distributed strain in optical fibers," IEEE Photonics Technology Letters 2(5), 352-354 (1990) 
3. T. Kurashima, T. Horiguchi and M. Tateda, "Distributed-temperature sensing using stimulated Brillouin scattering in optical silica fibers," Opt. Lett. 15(18), 1038-1040 (1990)

4. T. Horiguchi, T. Kurashima and M. Tateda, "Tensile strain dependence of Brillouin frequency shift in silica optical fibers," IEEE Photonics Technology Letters 1(5), 107-108 (1989)

5. M. Nikles, L. Thevenaz and P. A. Robert, "Brillouin gain spectrum characterization in single-mode optical fibers," Journal of Lightwave Technology 15(10), 1842-1851 (1997)

6. T. Horiguchi, K. Shimizu, T. Kurashima, M. Tateda and Y. Koyamada, "Development of a distributed sensing technique using Brillouin scattering," Journal of Lightwave Technology 13(7), 1296-1302 (1995)

7. M. Nikles, L. Thevenaz and P. A. Robert, "Simple distributed fiber sensor based on Brillouin gain spectrum analysis," Optics Letters 21(10), 758-760 (1996)

8. X. Bao, D. J. Webb and D. A. Jackson, "22-km distributed temperature sensor using Brillouin gain in an optical fiber," Opt. Lett. 18(7), 552-554 (1993)

9. A. Fellay, L. Thevenaz, M. Facchini, M. Nikles and P. Robert, "Distributed sensing using stimulated Brillouin scattering: towards ultimate resolution," in 12th International Conference on Optical Fiber Sensors. Technical Digest., pp. 324-327, Opt. Soc. America, Williamsburg, VA, USA (1997).

10. R. W. Boyd, Nonlinear Optics, Academic, New York (2003).

11. K. Hotate, "Measurement of Brillouin gain spectrum distribution along an optical fiber using a correlation-based technique-proposal, experiment and simulation," IEICE Transactions on Electronics E83-C(3), 405-411 (2000)

12. K. Hotate and M. Tanaka, "Distributed fiber Brillouin strain sensing with 1-cm spatial resolution by correlation-based continuous-wave technique," IEEE Photonics Technology Letters 14(2), 179-181 (2002)

13. X. Bao, A. Brown, M. DeMerchant and J. Smith, "Characterization of the Brillouin-loss spectrum of single-mode fibers by use of very short (\&lt;10-ns) pulses," Opt. Lett. 24(8), 510-512 (1999)

14. V. Lecoeuche, D. J. Webb, C. N. Pannell and D. A. Jackson, "Transient response in high-resolution Brillouin-based distributed sensing using probe pulses shorter than the acoustic relaxation time," Opt. Lett. 25(3), 156-158 (2000)

15. A. W. Brown, B. G. Colpitts and K. Brown, "Dark-Pulse Brillouin Optical Time-Domain Sensor With 20-mm Spatial Resolution," J. Lightwave Technol. 25(1), 381-386 (2007)

16. S. Foaleng-Mafang, J.-C. Beugnot and L. Thevenaz, "Optimized configuration for high resolution distributed sensing using Brillouin echoes," in 20th International Conference on Optical Fibre Sensors J. D. C. Jones, Ed., pp. 75032C75034, SPIE, Edinburgh, United Kingdom (2009).

17. L. Thevenaz and S. F. Mafang, "Distributed fiber sensing using Brillouin echoes," in 19th International Conference on Optical Fibre Sensors, pp. 70043N-70044, SPIE, Perth, WA, Australia (2008).

18. L. Thevenaz and J.-C. Beugnot, "General analytical model for distributed Brillouin sensors with sub-meter spatial resolution," in 20th International Conference on Optical Fibre Sensors J. D. C. Jones, Ed., pp. 75036A-75034, SPIE, Edinburgh, United Kingdom (2009).

19. W. Li, X. Bao, Y. Li and L. Chen, "Differential pulse-width pair BOTDA for high spatial resolution sensing," Opt. Express 16(26), 21616-21625 (2008)

20. K. Y. Song, W. Zou, Z. He and K. Hotate, "All-optical dynamic grating generation based on Brillouin scattering in polarization-maintaining fiber," Opt. Lett. 33(9), 926-928 (2008)

21. Y. Dong, X. Bao and L. Chen, "Distributed temperature sensing based on birefringence effect on transient Brillouin grating in a polarization-maintaining photonic crystal fiber," Opt. Lett. 34(17), 2590-2592 (2009)

22. K. Y. Song, W. Zou, Z. He and K. Hotate, "Optical time-domain measurement of Brillouin dynamic grating spectrum in a polarization-maintaining fiber," Opt. Lett. 34(9), 1381-1383 (2009)

23. Y. Dong, L. Chen and X. Bao, "Truly distributed birefringence measurement of polarization-maintaining fibers based on transient Brillouin grating," Opt. Lett. 35(2), 193-195 (2010)

24. W. Zou, Z. He, K.-Y. Song and K. Hotate, "Correlation-based distributed measurement of a dynamic grating spectrum generated in stimulated Brillouin scattering in a polarization-maintaining optical fiber," Opt. Lett. 34(7), 1126-1128 (2009)

25. W. Zou, Z. He and K. Hotate, "Demonstration of Brillouin Distributed Discrimination of Strain and Temperature Using a Polarization-Maintaining Optical Fiber," IEEE Photonics Technology Letters 22(8), 526-528 (2010)

26. K.-Y. Song, S. Chin, N. Primerov and L. Thevenaz, "Time-domain distributed fiber sensor with $1 \mathrm{~cm}$ spatial resolution based on Brillouin dynamic grating," Journal of Lightwave Technology 28, in press (2010). 